\title{
On the use of the thermal wave resonator cavity sensor for monitoring hydrocarbon vapors
}

J. A. P. Lima, E. Marín, M. G. da Silva, M. S. Sthel, S. L. Cardoso, D. F. Takeuti, C. Gatts, H. Vargas, C. E. Rezende, and L. C. M. Miranda

Citation: Review of Scientific Instruments 71, 2928 (2000); doi: 10.1063/1.1150712

View online: https://doi.org/10.1063/1.1150712

View Table of Contents: http://aip.scitation.org/toc/rsi/71/7

Published by the American Institute of Physics

\section{Articles you may be interested in}

Thermal-wave resonator cavity

Review of Scientific Instruments 66, 4999 (1995); 10.1063/1.1146123

A method for thermal diffusivity measurement in fluids

Review of Scientific Instruments 84, 104903 (2013); 10.1063/1.4824191

Application of the thermal wave resonator to the measurement of the thermal diffusivity of gas mixtures Review of Scientific Instruments 72, 1580 (2001); 10.1063/1.1334622

Thermal-wave resonator cavity design and measurements of the thermal diffusivity of liquids

Review of Scientific Instruments 71, 2933 (2000); 10.1063/1.1150713

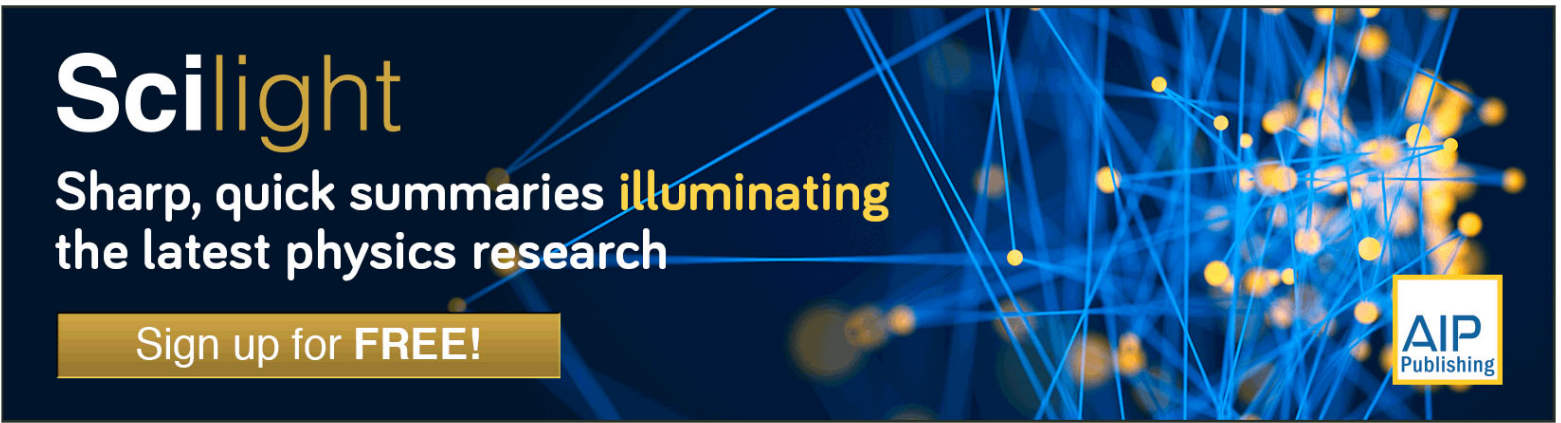




\title{
On the use of the thermal wave resonator cavity sensor for monitoring hydrocarbon vapors
}

\author{
J. A. P. Lima, E. Marín, a) M. G. da Silva, M. S. Sthel, S. L. Cardoso, D. F. Takeuti, \\ C. Gatts, and H. Vargas ${ }^{b)}$ \\ Centro de Ciência e Tecnología, Universidade Estadual do Norte Fluminense, Av. Alberto Lamego 2000, \\ 28015-620 Campos dos Goytacazes, RJ, Brazil \\ C. E. Rezende \\ Centro de Biología e Biotecnología, Universidade Estadual do Norte Fluminense, \\ Av. Alberto Lamego 2000, 28015-620 Campos dos Goytacazes, RJ, Brazil \\ L. C. M. Miranda \\ Departamento de Física, Universidade Estadual de Maringá, 87020-900 Maringá, PR, Brazil
}

(Received 22 November 1999; accepted for publication 7 April 2000)

A gas sensing device based on a thermal wave resonator cavity is outlined. It is experimentally tested by monitoring the presence of several hydrocarbon vapors in air via the measurement of the thermal diffusivity. It is also shown that its time-dependent response may be used to follow the vapor diffusion. It is shown that its characteristic response time is linearly correlated to the thermal diffusivity value of the mixture. The steps toward the development of a practical sensing device are further discussed. (C) 2000 American Institute of Physics. [S0034-6748(00)03107-5]

\section{INTRODUCTION}

During the past few decades, various different schemes for air monitoring have been developed and extensively discussed in the literature. ${ }^{1}$ Despite the important advantages of the traditional methods, such as spectroscopic techniques, there are still some drawbacks that have so far delayed their application for routine purposes. The instrumentation is often rather complex, requiring specially trained personnel able to face complicated data processing. On the other hand, there is a well-known expanding demand for reliable and precise measurement of the basic properties of the wide range of gases in use to date and/or generated by nature and modern industry, whose knowledge can lead scientists to a better understanding of the processes and phenomena governing this subject. The situation is particularly critical on the evaluation of thermal properties such as thermal diffusivity, a thermal transport parameter which determines the rate of heat diffusion in a medium and which can be considered as its fingerprint, for which few determinations have been reported, mostly related to measurements made in the late 1950 s. $^{2}$

To overcome this lack of information, and inspired by the pioneering work of Shen and Mandelis, ${ }^{3}$ a device based upon the generation and detection of thermal waves was developed. The proposed method is another application of the photothermal techniques, a field that has witnessed an enormous expansion in many directions as a result of its general applicability and adaptability to many areas of research. For a review on the applications of the photothermal techniques we refer to Refs. $4-6$. In the present paper the method is

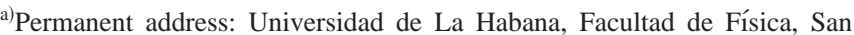
Lázaro y L, Vedado 10400, La Habana, Cuba.

b)Electronic mail: vargas@uenf.br
}

applied to the measurement of the thermal diffusivity of air contaminated with vapors of different liquid hydrocarbons of the paraffin family. These hydrocarbons are characterized by molecules forming a linear saturated chain of $\mathrm{C}-\mathrm{H}$ bonds given by the formula $\mathrm{C}_{n} \mathrm{H}_{2 n+2} .{ }^{7}$ In Sec. II we discuss the physical basis of the measuring method, our experimental setup, as well as the measuring methodology. The results of our measurements are presented and discussed in Sec. III.

\section{METHODOLOGY}

The instrument designed in our laboratories consists basically of a closed cell, suitable for gas exchange and control of ambient parameters, containing the gas under investigation, in which a thermal wave resonant cavity (TWRC) $)^{3}$ is enclosed. This cavity, of variable length, is formed between a thin $\mathrm{Al}$ foil and a pyroelectric temperature sensor. An intensity modulated light beam impinges on the black-painted external face of the $\mathrm{Al}$ foil, which acts as a light absorber. As a result of the absorption of the modulated incident light, the $\mathrm{Al}$ foil temperature fluctuates periodically, thereby launching a thermal wave into the gas-filled cavity. On striking the gas-Al and gas-sensor boundaries, the wave will be partially reflected and interference between the reflected and incident wave trains will set in.

The temperature rise at the pyroelectric surface, which results from the superposition of all arriving waves, can be estimated using the following model. Consider the situation depicted in Fig. 1. A gas region of thickness $L$ is sandwiched between the thin (15- $\mu \mathrm{m}$-thick) Al foil and a pyroelectric sensor [a 25- $\mu \mathrm{m}$-thick polyvinyledene difluoride (PVDF) film with $\mathrm{Al}$ metalized surfaces]. The outer surface of the $\mathrm{Al}$ film is heated by a light source of periodically modulated intensity $I_{0}[1+\cos (\omega t)] / 2$, where $I_{0}$ is the light source intensity before the modulation at the angular modulation fre- 


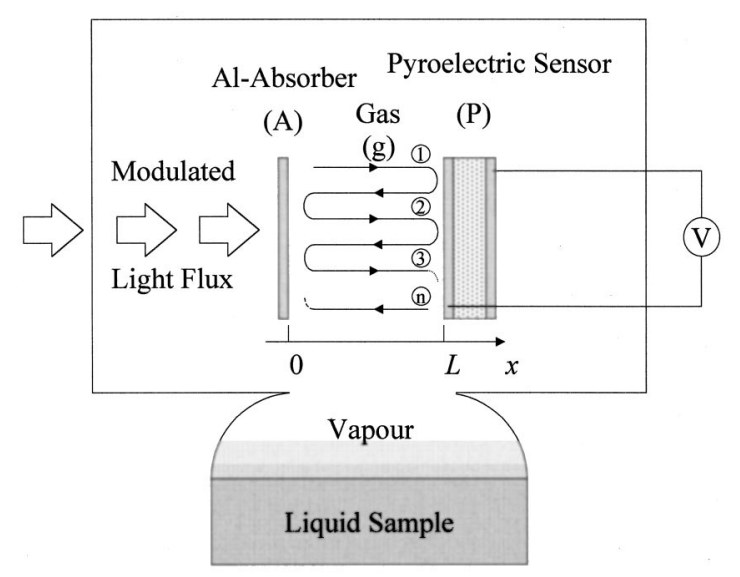

FIG. 1. Schematic view of the thermal wave resonator cavity sensor described in the text.

quency $\omega$ and $t$ is the time. The temperature distribution $T(x, t)$ within the gas region along the longitudinal $x$ coordinate, following the periodic heating of the $\mathrm{Al}$ foil, can be obtained by solving the heat diffusion equation with the boundary condition that the heat generated at the solid surface by light absorption is dissipated in the gas by diffusion. The solution of physical interest for applications in PT techniques is the one related to the time-dependent component of the light flux. If we assume this component, the general solution of the problem becomes ${ }^{6}$

$$
\begin{aligned}
T(x, t) & =\frac{I_{0}}{2 \epsilon_{g} \sqrt{\omega}} \exp (-q x) \exp (i \omega t) \\
& =\frac{I_{0}}{2 \epsilon_{g} \sqrt{\omega}} \exp (-x / \mu) \exp [-i(x / \mu-\pi / 4-\omega t)],
\end{aligned}
$$

where $\epsilon_{g}$ is the thermal effusivity of the gas, $q$ $=\left(i \omega / \alpha_{g}\right)^{1 / 2}, \alpha_{g}$ is the gas thermal diffusivity, $i=(-1)^{1 / 2}$, and $\mu=\left(2 \alpha_{g} / \omega\right)^{1 / 2}$ represents the thermal diffusion length in the gas of the thermal wave given by Eq. (1).

Thermal waves can be reflected at interfaces. ${ }^{6,8}$ If we assume that a plane wave propagates from a medium 1 to a medium 2, then for normal incidence the reflection coefficient at a boundary between the two media is given by

$$
R_{12}=\frac{1-b}{1+b}
$$

with $b=\epsilon_{2} / \epsilon_{1}$. This parameter may be regarded as being a measure of the thermal mismatch between the two media. Consider now the propagation of a plane thermal wave described by Eq. (1) through a gas (denoted by $g$ ) contained between the media $A$ (corresponding to the Al foil) and $P$ (corresponding to a PVDF sensor). The temperature at the surface $x=L$ is obtained by summing all the waves arriving at this point in the following way [for the sake of simplicity, from now on we will omit the term $\exp (i \omega t)$ in our deductions]:

$$
\begin{aligned}
T(L)= & T_{1}+T_{2}+\cdots+T_{n} \\
= & T_{0}\left[\exp (-q L)+R_{g A} R_{g P} \exp (-3 q L)\right. \\
& \left.+\cdots+\left(R_{g A} R_{g P}\right)^{n} \exp [-(2 n+1) q L]\right],
\end{aligned}
$$

where $T_{0}=I_{0} /\left(2 \epsilon_{g} \omega^{1 / 2}\right)$ is the gas temperature at $x=0$. The coefficients $R_{g A}$ and $R_{g P}$ are the reflection coefficients at the interfaces gas- $\mathrm{Al}$ and gas-PVDF, respectively, as given by Eq. (2). The variable $n$ takes positive integer values from 0 on. Equation (3) can be rewritten as

$$
\begin{aligned}
T(L) & =T_{0} \exp (-q L) \sum_{n=0}^{\infty}[\gamma \exp (-2 q L)]^{n} \\
& =T_{0} \exp (-q L) \sum_{n=0}^{\infty} r^{n},
\end{aligned}
$$

with $\gamma=R_{g A} R_{g P}$. The series $\sum_{n=0}^{\infty} r^{n}$, for $|r|<1$, is the geometric progression, which converges to the function $1 /(1-r) .{ }^{9}$ By definition $\gamma<1$ and $\exp (-q L)<1$, so that we get

$$
T(L)=\frac{T_{0} \exp (-q L)}{1-\gamma \exp (-2 q L)} .
$$

In the preceding discussion we have focused our attention on the physical aspects of the problem, giving a phenomenological explanation. A more rigorous treatment, leading to the same result when adapted to this special case, is given in detail elsewhere. ${ }^{10}$ The influence of heat dissipation by convection and radiation into the gas cavity was also analyzed before, ${ }^{11}$ and it was concluded that the main mechanism explaining the experimental results is based on heat diffusion.

The temperature rise given by Eq. (5) induces an electric field between the two opposite surfaces of the PVDF sensor. The resulting voltage $V$ is given by a complex function,

$$
V=V_{0}\left\langle T_{p}\right\rangle,
$$

where $V_{0}$ is a complex function containing the frequency dependence of the pyroelectric sensor signal and geometrical parameters characterizing the detection system, ${ }^{12}$ and $\left\langle T_{p}\right\rangle$ is the average temperature rise of the pyroelectric sensor, proportional to $T(L)$. We note from Eqs. (5) and (6) that the pyroelectric signal depends essentially on the thermal diffusivity and thermal effusivity of the gas, a fact that renders this technique quite suitable for the evaluation of these thermal properties as well as for the monitoring of dynamic processes in gas mixtures. The real (in-phase) and imaginary (in-quadrature) parts of $V$, as well as its amplitude and phase, can be determined by lock-in based detection. In the experimental setup, the sensor side of the cavity was assembled on a micrometer stage, which allows the scanning of a 4-mm-long cavity at a step resolution of $10 \mu \mathrm{m}$. Thermal waves were generated by the illumination, at normal incidence, of the $\mathrm{Al}$ foil by a $20 \mathrm{~mW}$ Meredith Instruments $\mathrm{He}-\mathrm{Ne}$ laser, mechanically modulated with a Model 650 EG\&G chopper at a frequency $f=\omega / 2 \pi=10 \mathrm{~Hz}$. The pyroelectric signal was measured using a model 5210 EG\&G lock-in amplifier interfaced to a personal computer. 

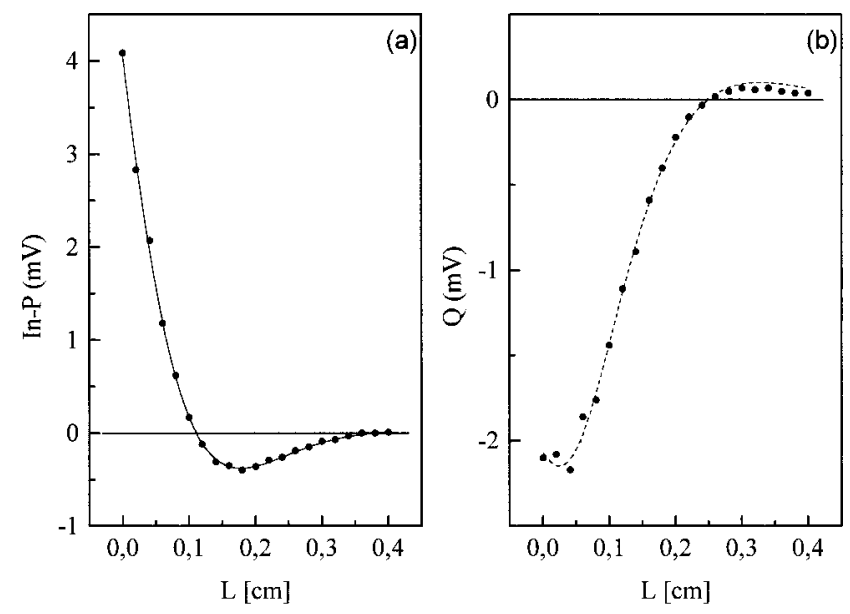

FIG. 2. In-phase (a) and quadrature (b) signals measured for air. The solid curves are the best-fit results to the real and imaginary parts of Eq. (6), respectively.

\section{EXPERIMENTAL RESULTS AND DISCUSSION}

In Fig. 2 we present the results we have obtained for the air-filled cell at ambient pressure and temperature. Figure 2(a) shows the in-phase (in-P) signal as a function of the cavity length $(L)$, whereas in Fig. 2(b) the quadrature $(Q)$ signal versus $L$ is plotted. The solid and dashed lines represent, respectively, the best fit of the experimental data to the real and imaginary parts of Eq. (6). The thermal diffusivity $\alpha$, present in the exponent of Eq. (5), was taken as adjustable parameter. The value we got for the thermal diffusivity of the air was $\alpha=0.244 \pm 0.007 \mathrm{~cm}^{2} / \mathrm{s}$, which is in good agreement with literature reported values. ${ }^{2}$

To further demonstrate the analytical potentialities of the proposed TWRC method we next performed a series of kinetic measurements with gas mixtures. At the bottom of the cell of total volume $210 \mathrm{~cm}^{3}$, a recipient containing approximately $1 \mathrm{~cm}^{3}$ of a liquid hydrocarbon sample is adapted (see Fig. 1). The vapor resulting from the hydrocarbon sample evaporation at ambient temperature diffuses into the air, filling the TWRC with an air-hydrocarbon gas mixture. In Fig. 3(a) we show a typical time evolution of the TWRC signal for different hydrocarbon samples for a fixed cavity length of $L=2 \mathrm{~mm}$. The observed time dependence of the pyroelectric signal is a consequence of the time evolution of the concentration of the gas mixtures due to the diffusion of hydrocarbon vapor molecules into the air. The thermal diffusivity of the resulting gas mixture varies with time, following the changes of the vapor concentration in air until saturation is reached. At this point, the vapor pressure of both components (air and hydrocarbon vapors) is equal, and the thermal diffusivity of the mixture takes a constant equilibrium value. The solid lines in Fig. 3(a) represent the data fitting to an exponential decay law. The values of the decay time $\tau$ obtained from the data fitting are plotted in Fig. 3(b), as a function of the number $N_{\mathrm{C}}$ of $\mathrm{C}$ atoms in the linear chains of the $\mathrm{C}-\mathrm{H}$ bonds of the samples. The solid lines in Fig. 3(b) represent the data linear regression. These results indicate that the decay time $\tau$ seems to be unique for a given airhydrocarbon mixture and may, accordingly, be used as a fingerprint of the gas mixture.
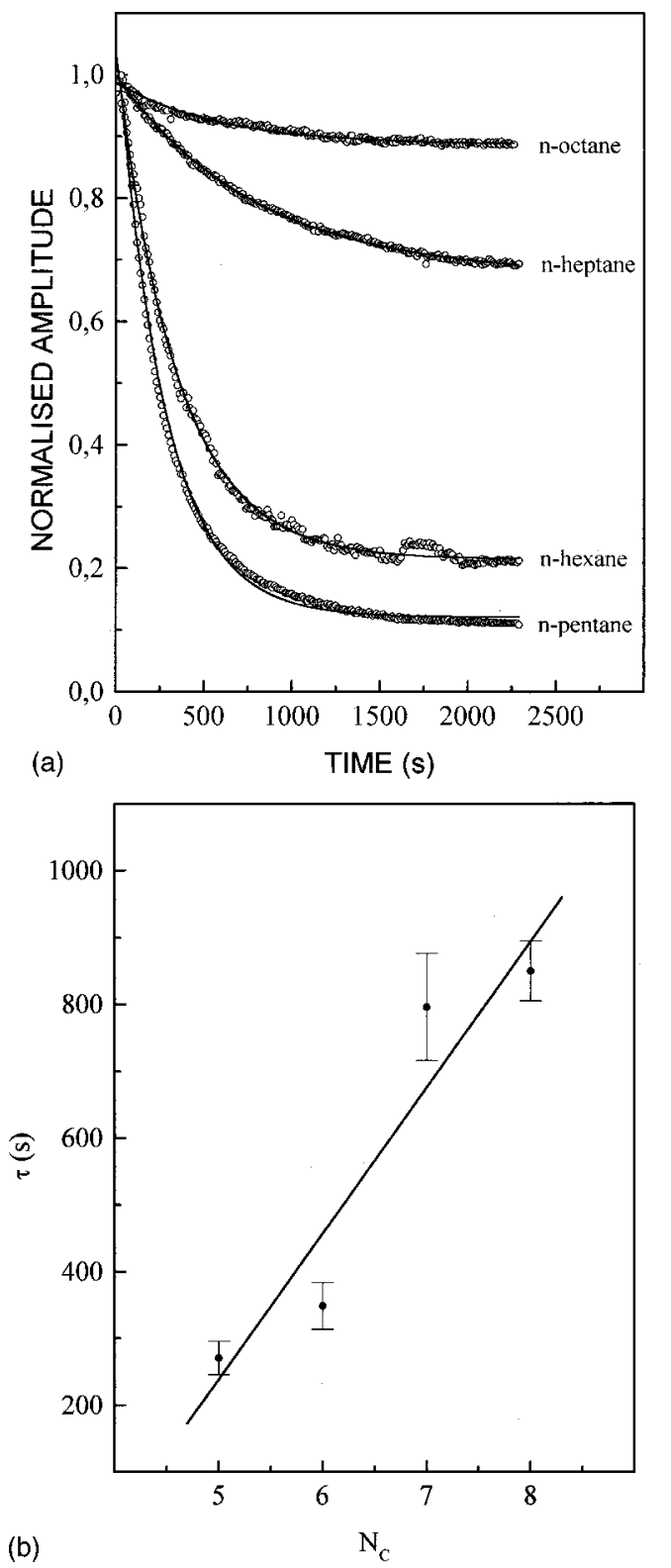

FIG. 3. (a) Time evolution (normalized signal as a function of time) of the TWRC signal corresponding to different hydrocarbon samples. The solid curves are the best-fit result of the data to an exponential decay law. (b) Decay time obtained from the fits represented in (a) as a function of the number of carbon atoms in the linear chain.

Next, as soon as the TWRC signal of the gas mixture stopped its time evolution, we performed the gas mixture thermal diffusivity measurements, following the same procedure as the one outlined previously for the case of air, namely, by recording the pyroelectric signal as a function of the cavity length, at fixed modulation frequency. In Table I we summarize the values we got for the thermal diffusivity values corresponding to the different air-hydrocarbon mixtures. Table I also presents the mole fraction of the hydrocarbons in each investigated mixture. In Fig. 4 we plot the corresponding values of $\alpha$ as a function of $N_{\mathrm{C}}$. As in the previous case of $\tau$, the solid line in Fig. 4 represents the data linear regression. We observe that the thermal diffusivity of the mixture corresponding to the $n$-octane hydrocarbon vapor has a higher value than those corresponding to the other va- 
TABLE I. Thermal diffusivity of the gas mixtures corresponding to the different hydrocarbons. For comparison, the measured thermal diffusivity of air was determined as $0.244 \mathrm{~cm}^{2} / \mathrm{s}$.

\begin{tabular}{lcc}
\hline \hline Hydrocarbon & $\begin{array}{c}\text { Hydrocarbon mole fraction } \\
\text { in the mixture }(\%)\end{array}$ & $\begin{array}{c}\text { Air-hydrocarbon mixture } \\
\text { thermal diffusivity }\left(\mathrm{cm}^{2} / \mathrm{s}\right)\end{array}$ \\
\hline$n$-pentane $\mathrm{C}_{5} \mathrm{H}_{12}$ & 40.1 & $0.069 \pm 0.002$ \\
$n$-hexane $\mathrm{C}_{6} \mathrm{H}_{14}$ & 16.9 & $0.112 \pm 0.002$ \\
$n$-heptane $\mathrm{C}_{7} \mathrm{H}_{16}$ & 5.98 & $0.148 \pm 0.003$ \\
$n$-octane $\mathrm{C}_{8} \mathrm{H}_{18}$ & 2.1 & $0.201 \pm 0.003$ \\
\hline \hline
\end{tabular}

pors. This behavior could be explained considering that the measured thermal diffusivity is in fact an effective value for the gas mixture. The concentration of $n$-octane vapor in the cell is lower than those of the other ones, therefore the measured thermal diffusivity in this case will be closer to that of the air. Conversely, the vapor concentration in air for the other hydrocarbons is higher, leading to a more pronounced variation in thermal diffusivity as compared to the value corresponding to air.

Finally, in Fig. 5 we plot the correlation between the measured values of $\alpha$ and $\tau$. This is an interesting result telling us that, at least for the present case of binary mixtures, these parameters are not only strongly correlated, but also that by measuring, say, $\tau$ in an unknown binary mixture we can determine the corresponding value of $\alpha$, and vice versa. That is, from the qualitative point of view, the abovementioned results indicate that both $\tau$ and $\alpha$ seem to be unique for each air-hydrocarbon mixture as the plots of both $\alpha$ and $\tau$ as a function of $N_{\mathrm{C}}$ indicate. To verify that this is actually the case we have next repeated these measurements of $\tau$ and $\alpha$ at several temperatures of the liquid hydrocarbon samples, that is, for different concentrations of the airhydrocarbon mixtures. The different temperatures were achieved using a Neslab RTE-111 constant temperature bath. The results of these measurements for the thermal diffusivity

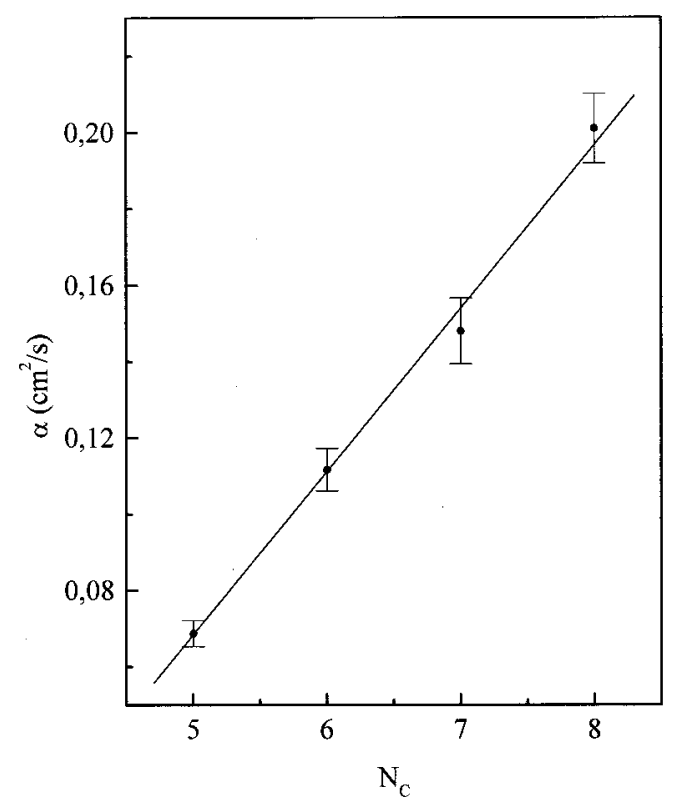

FIG. 4. Thermal diffusivity as a function of the number of carbon atoms in the linear chain.

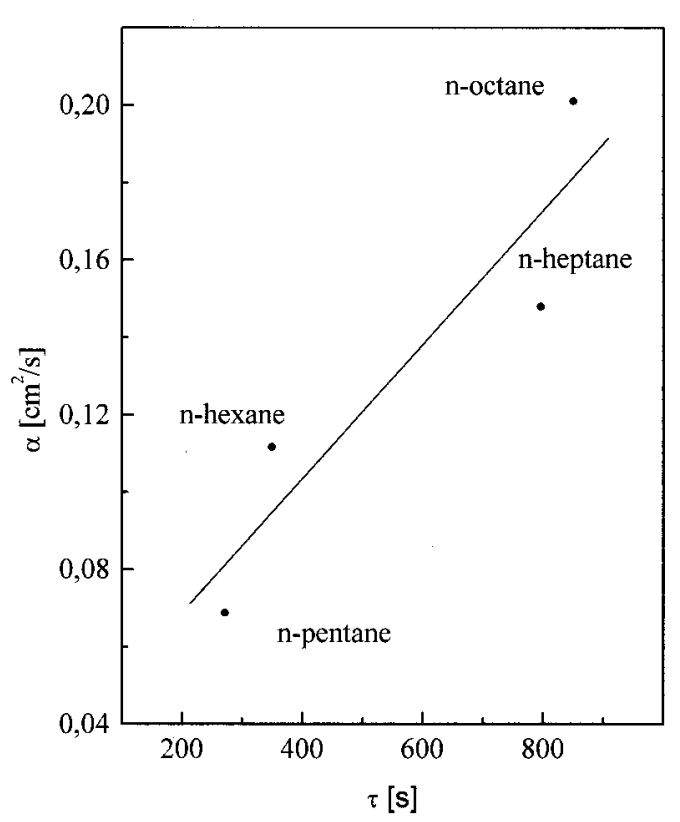

FIG. 5. Thermal diffusivity vs decay times. The solid line is a data linear regression.

are shown in Fig. 6. The corresponding curve for $n$-octane was not measured, because the high temperatures necessary to obtain appreciable changes in the vapor concentration have led to uncontrollable noise in our measurements. Similar curves were obtained for $\tau$. These plots confirm the hypothesis that the decay time and the thermal diffusivity are indeed unique for each air-hydrocarbon mixture, exhibiting no intersection between the characteristic curves for each mixture.

The above-mentioned results lead us to propose a new

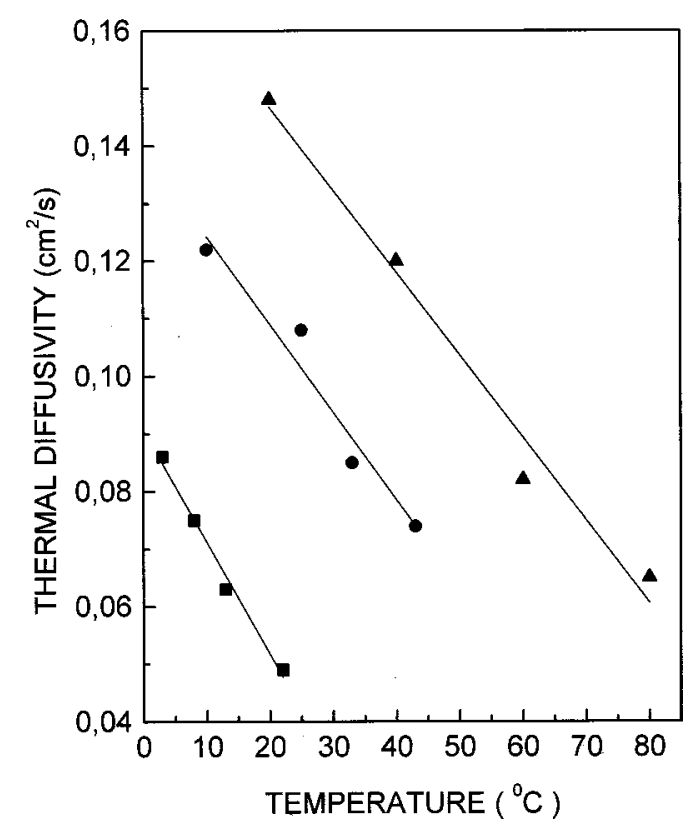

FIG. 6. Thermal diffusivity as a function of the temperature for several air-hydrocarbon mixtures. The squares, circles, and up-triangle correspond to $n$-pentane, $n$-hexane, and $n$-heptane, respectively. The solid lines are the best linear fits of the data. 
scheme for the development of gas analyzers, based upon the use of a TWRC. The main advantage of the proposed technique is that it can be applied to the characterization of both light absorbing gases and optically transparent gases. The traditional methods used in gas analysis are based on the absorption of certain characteristic laser lines by the gas molecules, typically in the infrared range of the electromagnetic spectrum, thus leading to the use of expensive signal generation and detection schemes. The results presented here open the possibility to perform routine, accurate measurement of thermal diffusivity of gases and, consequently, to monitor environmental air pollution related phenomena. Furthermore, a wide range of applications, including determination of respiration products (soils and plants) can also be envisaged. The results obtained for the analysis of contaminated air points to the possibility of detecting the presence of pollutants in ambient air through the measurement of its thermal diffusivity.

For the specific case of air-hydrocarbon mixture, the results also show that the measurements of $\tau$ and $\alpha$ may indeed be used to monitor the presence of hydrocarbons in the air. The procedure in this case is clear. One should tabulate the characteristic curves of the several air-hydrocarbon mixtures, at different concentrations, and compare the corresponding $\tau$ and $\alpha$ value obtained from the TWRC signal for the gas sample under test with the corresponding tabulated values. In terms of practical applications, one immediate use of these results may be the monitoring of hydrocarbon leakage from storage tanks.

\section{ACKNOWLEDGMENTS}

The authors greatly acknowledge L. A. M. Mireilles and F. R. da Silva for technical assistance. E. M. also thanks the postdoctoral program of the Centro Latinoamericano de Física (CLAF). This work was partially supported by CNPq and ANP (Brazil).

${ }^{1}$ M. W. Siegrist, AIR Monitoring by Spectroscopic Techniques, Chemical Analysis Vol. 127 (Wiley, New York, 1994).

${ }^{2}$ Y. S. Touloukian, R. W. Powell, Y. C. Ho, and M. C. Nocalasu, Thermal Diffusivity (Plenum, New York, 1973).

${ }^{3}$ J. Shen and A. Mandelis, Rev. Sci. Instrum. 66, 4999 (1995).

${ }^{4}$ A. Rosencwaig, Photoacoustics and Photoacoustic Spectroscopy (Wiley, New York, 1980).

${ }^{5}$ H. Vargas and L. C. M. Miranda, Phys. Rep. 161, 43 (1988).

${ }^{6}$ D. P. Almond and J. Patel, Photothermal Science and Techniques (Chapman and Hall, London, 1996).

${ }^{7}$ G. J. Van Wylen and R. E. Sonntag, Fundamentals of Classical Thermodynamics (Wiley, New York, 1985), Chap. 12.

${ }^{8}$ B. K. Bein and J. Pelzl, in Plasma Diagnostics, Surface Analysis and Interactions (Academic, New York, 1989).

${ }^{9}$ I. S. Gradshteyn, I. M. Ryzhik, and A. Jeffrey, Table of Integrals, Series and Products (Academic, New York, 1994).

${ }^{10}$ A. Mandelis, J. Vanniasinkam, S. Budhudu, A. Othonos, and M. Kokta, Phys. Rev. B 48, 6808 (1993).

${ }^{11}$ J. Shen, A. Mandelis, and H. Tsai, Rev. Sci. Instrum. 69, 197 (1998).

${ }^{12}$ A. Van der Ziel and S. T. Liu, J. Appl. Phys. 43, 4266 (1972). 